\title{
CONTROLE DOS PARÂMETROS DE SECAGEM NUMA INSTALAÇÃO EXPERIMENTAL UTILIZANDO LÓGICA DIFUSA
}

\author{
CAMILA N. BOERI ${ }^{1,2}$, FERNANDO J. NETO DA SILVA ${ }^{1}$, JORGE A. F. FERREIRA ${ }^{1}$.
}

\author{
1. Centro de Tecnologia Mecânica e Automação, Departamento de Engenharia Mecânica, \\ Universidade de Aveiro - Aveiro - Portugal \\ E-mails: camilaboeri@ua.pt, fneto@ua.pt, jaff@ua.pt
}

\author{
2. Departamento de Ciências Exatas e da Terra, Universidade Regional Integrada do Alto Uruguai e das \\ Missões - Campus Frederico Westphalen \\ Rua Assis Brasil, 709 - Caixa Postal: 184 - CEP 98400-000 - Frederico Westphalen - RS
}

\begin{abstract}
The contribution of this paper is to propose a strategy for nonlinear control, consisting of four fuzzy controllers: proportional and derivative controllers diffuse for a dehumidifier, a humidifier, a set of electrical resistances and a centrifugal blower used in the convective drying experimental installation. The results were compared with those obtained previously using a linear PID controller optimized. The objective of this research is to find the optimal conditions for salted codfish drying. The drying experimental tests were performed for the following operating conditions: air temperature of $15,18,20$ and $23{ }^{\circ} \mathrm{C}$, relative humidities of $40,45,50,55,60,65$ and $70 \%$ and air velocities equal to 1.5 and $2.5 \mathrm{~m} / \mathrm{s}$. The limitations of the experimental setup restrict values to a minimum relative humidity of $40 \%$. The air velocity values are within the range used for the drying of fish. Were parameterize a group of fuzzy sets, to ensure a high performance controller without need for the development of a mathematical model of the drying process. The results showed a better performance for the fuzzy controller compared to PID control.
\end{abstract}

Keywords—Control, Fuzzy Logic, Optimization, Drying

\begin{abstract}
Resumo- A contribuição deste artigo é a proposta de uma estratégia de controle não linear, composto por quatro controladores difusos: Controladores difusos proporcionais e derivativos para um desumificador, um umidificador, um conjunto de resistências elétricas e um ventilador utilizados na instalação laboratorial de secagem convectiva. Os resultados foram comparados com os obtidos anteriormente através de um controlador linear PID otimizado. O objetivo desta pesquisa é encontrar as condições ótimas para a secagem de bacalhau salgado verde. Os ensaios experimentais de secagem foram realizados para as seguintes condições operacionais: temperaturas do ar de $15,18,20$ e $23^{\circ} \mathrm{C}$; umidades relativas de 40, 45, 50, 55, 60, 65 e $70 \%$ e velocidades do ar iguais a 1,5 e $2,5 \mathrm{~m} / \mathrm{s}$. As limitações da instalação experimental restringem os valores de umidade relativa a um mínimo de $40 \%$. Os valores da velocidade do ar estão dentro do intervalo utilizado para a secagem de peixe. Foram parametrizados um grupo de conjuntos difusos, de forma a assegurar um alto desempenho do controlador, sem necessidade do desenvolvimento do modelo matemático do processo de secagem. A análise dos resultados mostrou um melhor desempenho para o controlador difuso quando comparado ao controle PID.
\end{abstract}

Palavras-chave— Controle, Lógica Difusa, Otimização, Secagem

\section{Introdução}

Um aspecto importante no que diz respeito à otimização das operações de secagem é o controle do seu processo. As barreiras principais à melhoria da eficiência energética do processo de secagem incluem a falta da compreensão dos fenômenos básicos que lhe estão associados e as limitações do equipamento de medida associado à operação de secadores.

A correta definição de procedimentos de secagem de uma vasta gama de produtos tem-se revelado crucial na minimização energética e na garantia da qualidade do produto final (Murugesan et al., 2002), o que justifica a necessidade do estudo dos procedimentos de secagem.

A secagem é um dos processamentos mais caros devido às exigências energéticas para movimentação e aquecimento do ar. $\mathrm{O}$ uso de estratégias de controle utilizando a combinação dos parâmetros envolvidos num processo de secagem visando a sua otimização representa, em termos práticos, redução de custos, um aumento da qualidade do produto, um menor tempo para execução de tarefas e uma maior eficiência energética.

No entanto, e devido à complexidade do seu comportamento dinâmico, o controle da secagem é provavelmente uma das áreas menos estudadas. Para esta ausência muito contribui a complexidade da modelação dinâmica, devido à natureza das equações diferenciais parciais altamente não lineares utilizadas na descrição de transferência de calor e massa, envolvendo coeficientes de transporte e propriedades termo-físicas fortemente relacionadas com a temperatura e teor de umidade do material. Justificase, assim, o estudo de estratégias de controle em processos de secagem visando a economia de energia e qualidade do produto.

De acordo com Gou et al. (2005), em instalações complexas, nomeadamente em tecnologia de 
secagem, é muitas vezes impossível formular um modelo físico-químico do processo, pois deve incluir-se os fenômenos de transferência de calor e de massa, que são caracterizados por uma transferência complexa de massa e energia. No entanto, através de regras e conjuntos difusos, é possível modelar qualquer função não linear e executar qualquer ação de controle não linear.

A lógica difusa é apropriada para a modelação e controle de sistemas complexos altamente não lineares, porque é um sistema formal que permite imitar a forma como a mente humana lida com a imprecisão. Assim, sistemas de controle difuso são amplamente utilizados para o controle de experimentos de secagem.

Segundo Azevedo et al. (2000), a base dos sistemas difusos é a teoria dos conjuntos fuzzy. Estes conjuntos são uma extensão dos conjuntos convencionais, os quais permitem somente que elementos sejam verdadeiros ou falsos. Os conjuntos difusos permitem que o seus elementos possuam um certo grau de pertença associado, sendo esta propriedade conhecida como multivalência. Isto permite a aproximação ao mundo real, que não é bivalente, com um vasto número de opções ao invés de somente duas. A lógica difusa permite trabalhar com tais incertezas de fenômenos naturais de forma rigorosa e sistemática.

De acordo com Olsson e Newell (1999), a grande vantagem da utilização da técnica de controle recorrendo à lógica difusa é o fato de aproveitar a experiência do utilizador facilitando a interação operador-máquina ao utilizar valores linguísticos, próprios do raciocínio humano. Para processos nos quais existe uma grande experiência operacional e para processos complexos ou de modelos demasiado complexos e não lineares, a lógica difusa apresentase como uma boa alternativa.

Conforme Feng et al. (2002), o controle difuso aplica, através de algoritmos, o conhecimento humano e pericial na solução de problemas com incertezas, não linearidades e atrasos de transporte. Uma vantagem do método é que o mesmo não precisa de inicialmente ser modelado matematicamente.

Neste trabalho, são descritos os ensaios em malha aberta realizados e o projeto e desenvolvimento dos controladores difusos para o umidificador, desumidificador, resistências elétricas e ventilador, bem como os resultados experimentais obtidos para o controle dos parâmetros de secagem numa instalação experimental convectiva. Os objetivos pretendidos com este controle são estabilizar os parâmetros dentro da instalação, reduzindo os erros entre os valores desejados e os obtidos para a temperatura, umidade relativa e velocidade do ar de secagem.

\section{Materiais e Métodos}

\subsection{Dinâmica do processo - Ensaios em malha aberta}

Para encontrar a melhor estratégia de controle é importante conhecer a dinâmica da máquina de secagem. Esta dinâmica mostra como é a variação do processo no tempo, as suas não-linearidades, a interdependência dos parâmetros de secagem e a influência da variação do sinal de controle no comportamento da dinâmica da temperatura, umidade e velocidade.

Estas não-linearidades foram estudadas através de um conjunto de procedimentos experimentais, em que:

(1) Em DC foi aplicado um sinal em rampa enquanto que R12C, R34C e UC foram mantidos constantes;

(2) DC foi projetado para apresentar uma resposta em degrau, enquanto que $\mathrm{R} 12 \mathrm{C}, \mathrm{R} 34 \mathrm{C}$ e $\mathrm{UC}$ foram mantidos constantes;

(3) UC foi projetado para apresentar uma resposta em degrau, enquanto que R12C, R34C e DC foram mantidos constantes;

(4) R12C, R34C foram projetados para apresentarem uma resposta em degrau, enquanto que DC e UC foram mantidos constantes;

(5) DC e UC foram projetados para apresentarem uma resposta em degrau enquanto que R12C, R34C foram mantidos constantes;

(6) $\mathrm{DC}$ e R12C, R34C foram projetados para apresentarem uma resposta em degrau, enquanto que UC foi mantido constante;

(7) R12C, R34C e UC foram projetados para apresentarem uma resposta em degrau enquanto que DC foi mantido constante;

(8) R12C, R34C, DC e UC foram projetados para apresentarem uma resposta em degrau.

Para este conjunto de ensaios em malha aberta, foram definidos dois pontos de operação inicial:

(1) $\mathrm{TR} \approx 24^{\circ} \mathrm{C}$ e $\mathrm{RHR} \approx 96 \%$;

(2) $\mathrm{TR} \approx 49^{\circ} \mathrm{C}$ e $\mathrm{RHR} \approx 10 \%$.

Ressalta-se que o desumidificador e o ventilador centrífugo foram mantidos em cerca de $50 \%$ da sua capacidade máxima, quando não estavam a ser controlados.

\subsection{Projeto dos controladores}

A lógica difusa é aplicada com sucesso no controle do processo em si, ou seja, no controle dos valores medidos. As vantagens da lógica difusa são óbvias quando está em causa o controle de um número de valores mutuamente dependentes, influenciados pelas mesmas variáveis de controle. Dessa forma, a lógica difusa permite que os sinais de erro de todos os valores controlados sejam levados em conta ao mesmo tempo, e, com base em regras difusas, obtidas através da investigação do processo controlado em diferentes condições de operação, são geradas as 
saídas que permitem trazer o sistema para a condição desejada.

Assim, um sistema de controle difuso é proposto para o controle de experimentos de secagem. Aqui é feita a proposta de uma estratégia de controle não linear composto por quatro controladores difusos: Controladores difusos proporcionais e derivativos para o Desumidificador (FDC-PD), Umidificador (FHRC-PD), Resistências Elétricas (FERC-PD) e Ventilador (FBC-PD).

De acordo com a metodologia baseada em Lógica Difusa, foram utilizados:

(1) Mecanismo de inferência do tipo "Mamdani";

(2) Método de implicação do tipo "minimum":

(3) Método de agregação do tipo "maximum":

(4) Método de desfuzificação do tipo centro de gravidade (COG):

Os controladores baseados em lógica difusa foram otimizados manualmente. Para a implementação, foi utilizado o software Matlab®, através das funções do Fuzzy Logic Toolbox's.

$\mathrm{O}$ esquema geral é mostrado na figura 1:

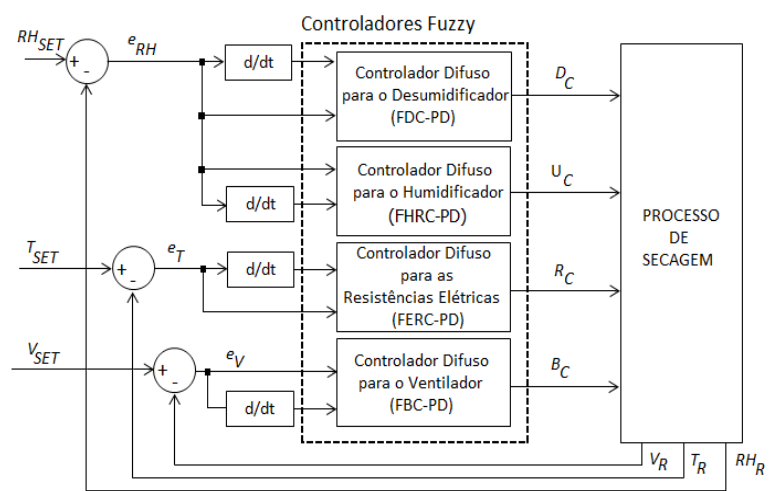

Figura 1: Esquema do controlo difuso

Em que:

BC Sinal do controlador para o ventilador centrífugo

DC Sinal do controlador para o desumidificador

eRH Erro da umidade relativa

eT Erro da temperatura

ev Erro da velocidade

FBC-PD Controlador difuso proporcional e derivativo para o ventilador centrífugo

FDC-PD Controlador difuso proporcional e derivativo para o desumidificador

FERC-PD Controlador difuso proporcional e derivativo para as resistências elétricas

FHRC-PD Controlador difuso proporcional e derivativo para o umidificador

$\mathrm{RC}$ Sinal do controlador para as resistências elétricas

RHR Umidade relativa real

RHSET Umidade relativa desejada

TR Temperatura real

TSET Temperatura desejada

UC Sinal do controlador para o humidificador

vR Velocidade real

vSET Velocidade desejada

\subsection{Controlador difuso para o umidificador}

Para o controle do humidificador foi desenvolvido um controlador proporcional e derivativo. Este controlador executa uma ação não-linear do erro atual e futuro para mapear a ação de controle da umidade relativa. Dezesseis conjuntos difusos e quarenta e cinco regras foram considerados para a concepção do mapeamento entre a entrada e a saída.

\subsection{Controlador difuso para o desumidificador}

Para o controle do desumidificador, foi projetado um controlador que define o mapeamento proporcional e derivativo da umidade relativa, através da ação nãolinear do erro atual e futuro. Dezenove conjuntos difusos e cinquenta e quatro regras foram definidos para o comportamento não-linear entre a entrada e a saída do controlador.

\subsection{Controlador difuso para as resistências elétricas}

Este controlador define a ação proporcional e derivativa do controle da temperatura do ar. Onze conjuntos difusos e nove regras foram concebidas para mapear a relação entre entrada e saída.

\subsection{Controlador difuso para o ventilador}

Este controlador realiza um mapeamento não-linear da ação de controle da velocidade do ar. Nove conjuntos difusos e nove regras foram parametrizados para caracterizar o comportamento utilizando o erro da velocidade atual e futura e a sua derivada.

\section{Resultados e Discussões}

\subsection{Ensaios em malha aberta}

Quatro conjuntos de resultados dos ensaios em malha aberta são mostrados nas figuras 2 a 5 . Convém ressaltar que o efeito da entrada em ação do desumidificador é duplo: o princípio de funcionamento do desumidificador baseia-se na redução da temperatura do ar e na promoção da condensação do vapor de água nas paredes frias do permutador da máquina frigorífica. Assim, o acionamento do desumidificador provoca invariavelmente a redução da temperatura enquanto a ação contrária provoca o aumento da temperatura. 


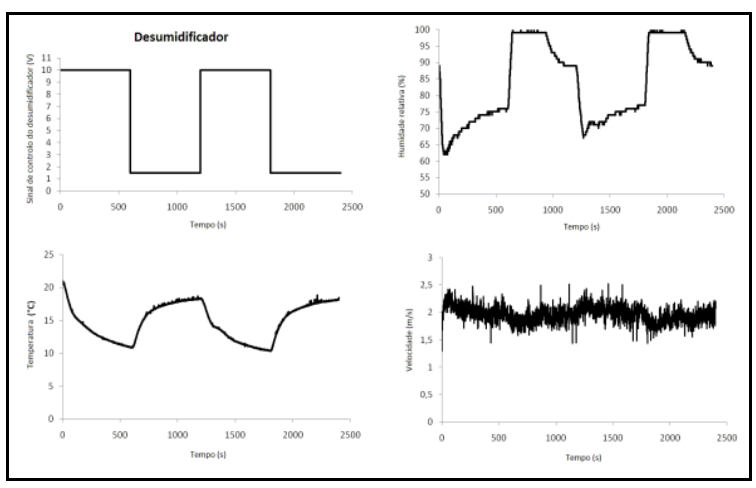

Figura 2: Dinâmica dos parâmetros do processo de secagem com a aplicação de sinal de comando variável ao desumidificador

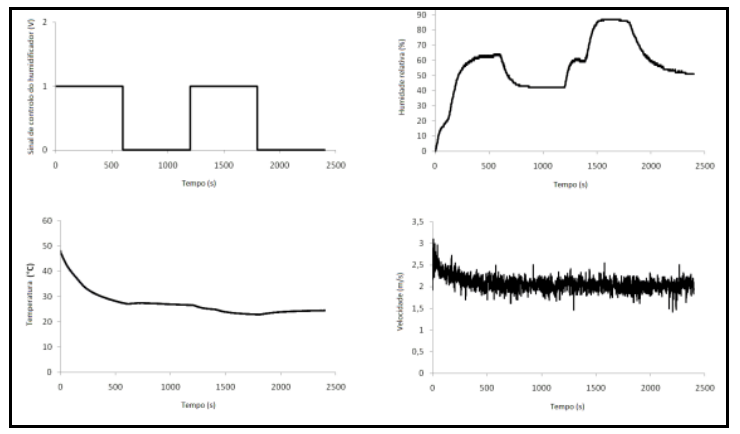

Figura 3: Dinâmica dos parâmetros do processo de secagem com a aplicação de uma onda quadrada ao umidificador

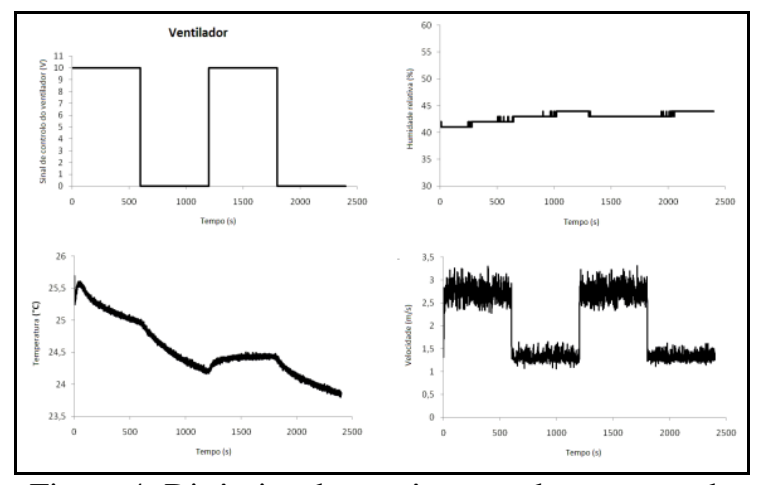

Figura 4: Dinâmica dos parâmetros do processo de secagem quando é aplicado um sinal em onda quadrada ao comando do ventilador

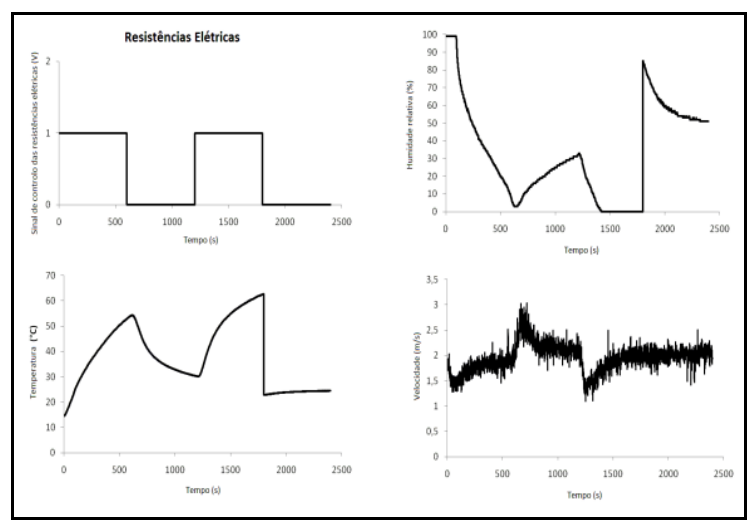

Figura 5: Dinâmica dos parâmetros do processo de secagem quando foi aplicado um sinal em onda quadrada ao sistema de comando das resistências de aquecimento
3.2 Controlador difuso para o umidificador (FHRCPD)

Parametrizou-se o controlador difuso para o umidificador definindo-se: duas entradas (erro da umidade relativa e a derivada do erro da umidade relativa); uma única saída (atuação da eletroválvula); cinco funções de pertença associadas aos valores linguísticos no intervalo $[-1,1]$ para a entrada relativa ao erro da umidade; nove funções de pertença associadas aos valores linguísticos no intervalo [-1, 1] para a entrada relativa à derivada do erro da umidade; duas funções de pertença associadas aos dois valores lingüísticos $(0$ e 1$)$ da saída. Foram estabelecidas 45 regras para o controlador difuso com saída on/off para o umidificador, aplicando-se um sistema de inferência do tipo Mamdani, em que se definiram os métodos de implicação, agregação e desfuzificação do tipo mínimo, máximo e centro de gravidade, respectivamente. A figura 6 apresenta a superfície de resposta do controle difuso para o umidificador.

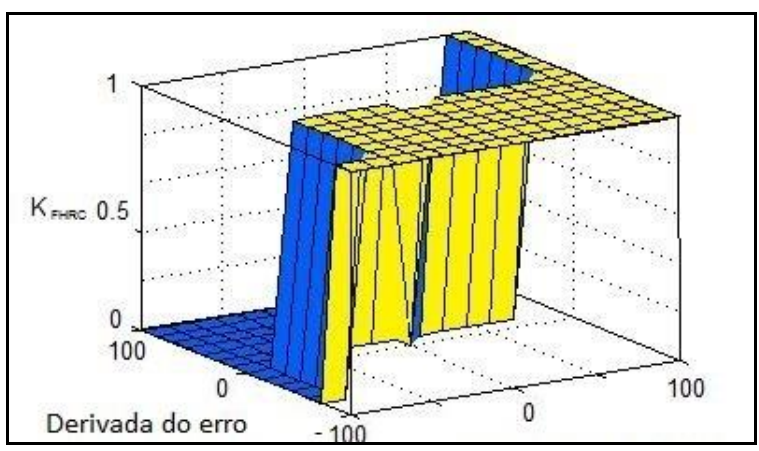

Figura 6: Superfície de saída do controlador difuso para o umidificador

\subsection{Controlador difuso para o desumidificador $(F D C-P D)$}

Parametrizou-se o controlador difuso para o desumidificador definindo-se: duas entradas (erro da umidade relativa e a derivada do erro da umidade relativa); uma única saída (atuação do compressor); seis funções de pertença associadas aos valores lingüísticos no intervalo [-1 1] para a entrada relativa ao erro da umidade; nove funções de pertença associadas aos valores linguísticos no intervalo [-1 1$]$ para a entrada relativa à derivada do erro da umidade; quatro funções de pertença associadas aos valores lingüísticos no intervalo [0 1 l $]$ da saída. Foram estabelecidas 54 regras para o controlador difuso para o desumidificador, aplicando-se um sistema de inferência do tipo Mamdani, em que se definiram os métodos de implicação, agregação e desfuzificação do tipo mínimo, máximo e centro de gravidade, respectivamente. A figura 7 apresenta a superfície de resposta do controle difuso para o desumidificador. 


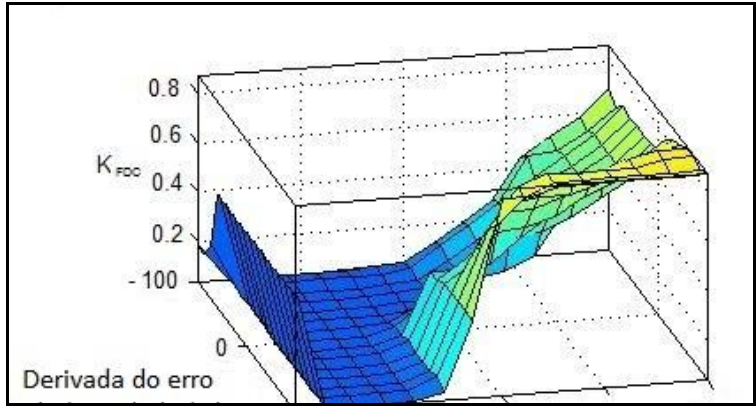

Figura 7: Superfície de saída do controlador difuso para o desumidificador

\subsection{Controlador difuso para as resistências elétricas (FERC-PD)}

Parametrizou-se o controlador difuso para as resistências elétricas definindo-se: duas entradas (erro da temperatura e a derivada do erro da temperatura); uma única saída (atuação das resistências elétricas); três funções de pertença associadas aos valores lingüísticos no intervalo [-1 1] para a entrada relativa ao erro da temperatura; três funções de pertença associadas aos valores linguísticos no intervalo [-1 1 ] para a entrada relativa à derivada do erro da temperatura; cinco funções de pertença associadas aos valores lingüísticos no intervalo [-1 1] da saída. Foram estabelecidas 9 regras para o controlador difuso para as resistências elétricas, aplicando-se um sistema de inferência do tipo Mamdani, em que se definiram os métodos de implicação, agregação e desfuzificação do tipo mínimo, máximo e centro de gravidade, respectivamente. As resistências são atuadas a partir de um sinal em PWM (pulse width modulation). Isto permite, muito embora seja um sinal on/off, variar a energia fornecida às resistências de uma forma proporcional ao sinal de controle (saída do controlador). A figura 8 apresenta a superfície de resposta do controlo difuso para as resistências elétricas.

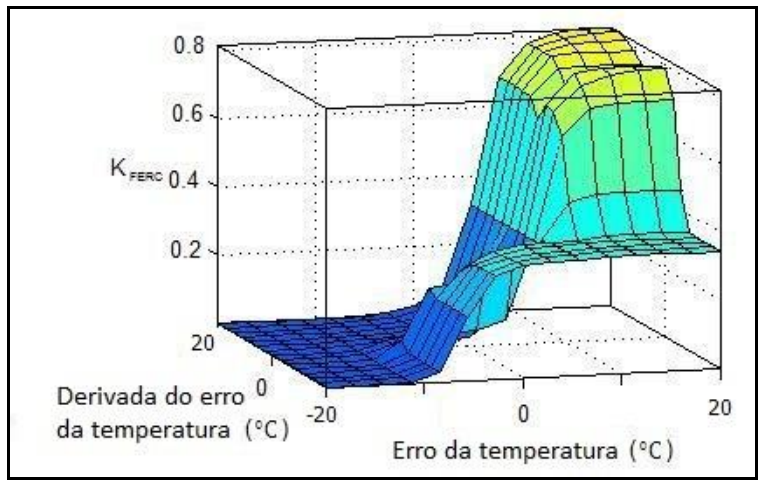

Figura 8: Superfície de saída do controlador difuso para as resistências elétricas

\subsection{Controlador difuso para o ventilador (FBC-PD)}

Parametrizou-se o controlador difuso para o ventilador definindo-se: duas entradas (erro da velocidade e a derivada do erro da velocidade); uma única saída (atuação do ventilador); três funções de pertença associadas aos valores lingüísticos no intervalo $[-1,1]$ para a entrada relativa ao erro da velocidade; três funções de pertença associadas aos valores linguísticos no intervalo $[-1,1]$ para a entrada relativa à derivada do erro da velocidade; três funções de pertença associadas aos valores linguísticos no intervalo $[-1,1]$ da saída. Foram estabelecidas 9 regras para o controlador difuso para o ventilador, aplicando-se um sistema de inferência do tipo Mamdani, em que se definiram os métodos de implicação, agregação e desfuzificação do tipo mínimo, máximo e centro de gravidade, respectivamente. A figura 9 apresenta a superfície de resposta do controle difuso para o ventilador.

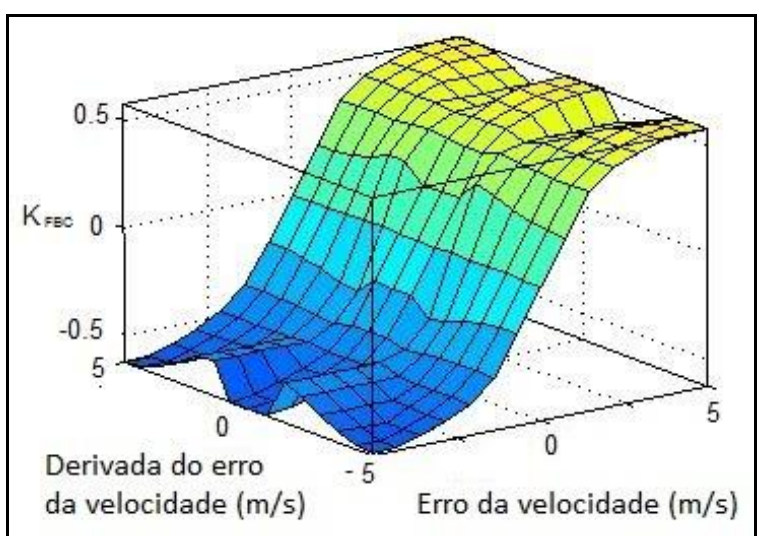

Figura 9: Superfície de saída do controlador difuso para o ventilador

\subsection{Resultados experimentais do controle dos parâmetros de secagem}

Alguns dos resultados obtidos para o controle da umidade relativa, temperatura e velocidade são mostrados nas figuras 10 a 13 .

Os ensaios foram realizados controlando-se simultaneamente os três parâmetros dentro da instalação, ou seja, a partir da atuação dos quatro controladores desenvolvidos (umidificador, desumidificador, resistências elétricas e ventilador).

Os resultados obtidos a partir do controle difuso foram comparados aos obtidos anteriormente com o controle PID, que existia na instalação. A análise do desempenho dos controladores mostrou melhores resultados para o controle difuso: a média dos erros absolutos foram inferiores a 2,79\% para o controle da umidade relativa e $0,349^{\circ} \mathrm{C}$ para o controle da temperatura, respectivamente, cerca de 3,93 e 3,71 vezes menor que os resultados experimentais encontrados por meio do controle PID. 


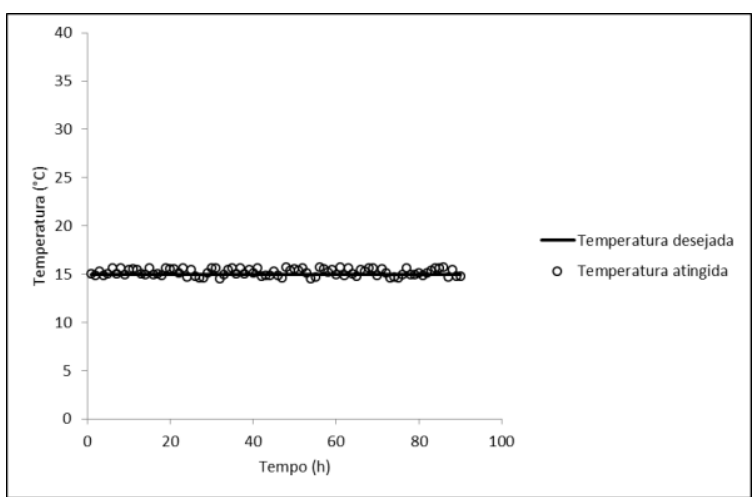

Figura 10: Variação da temperatura ao longo do tempo - Ensaio: $\mathrm{T}=15^{\circ} \mathrm{C} ; \mathrm{HR}=40 \%$; $=2 \mathrm{~m} / \mathrm{s}$

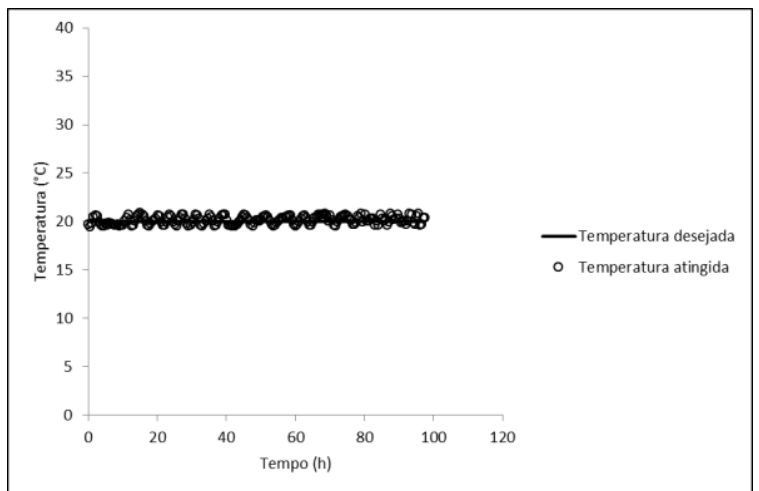

Figura 11: Variação da temperatura ao longo do tempo - Ensaio: $\mathrm{T}=20^{\circ} \mathrm{C} ; \mathrm{HR}=50 \%$; $=2 \mathrm{~m} / \mathrm{s}$

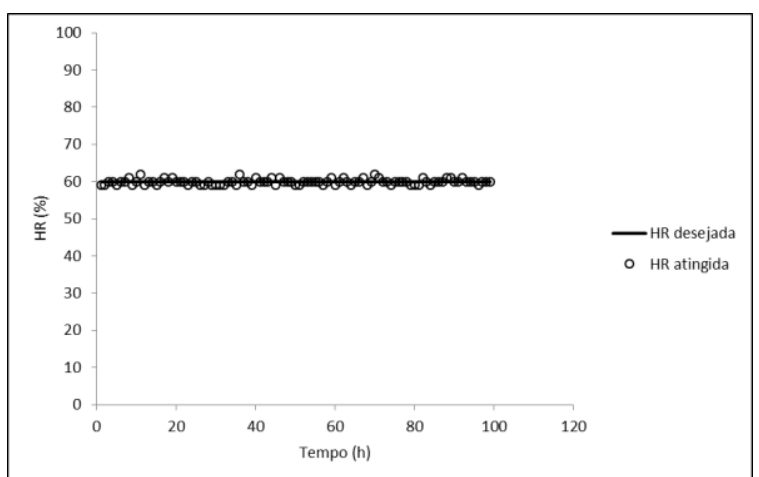

Figura 12: Variação da umidade relativa ao longo do tempo - Ensaio: $\mathrm{T}=20^{\circ} \mathrm{C} ; \mathrm{HR}=60 \% ; \mathrm{v}=1,5 \mathrm{~m} / \mathrm{s}$

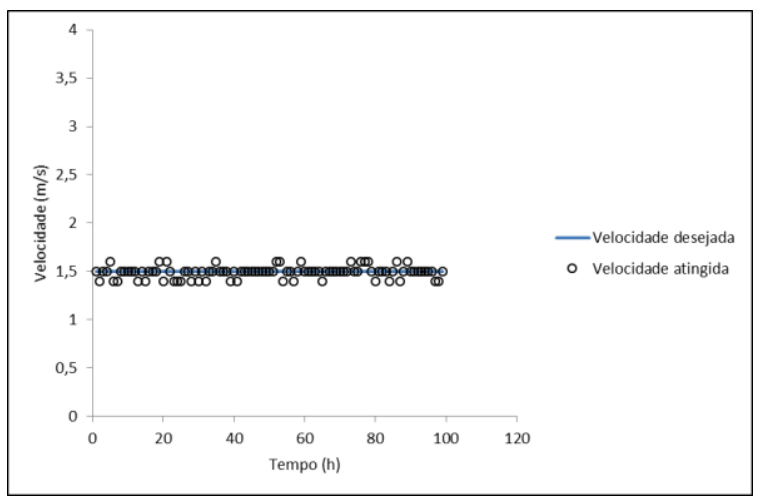

Figura 13: Variação da velocidade ao longo do tempo - Ensaio: $\mathrm{T}=20^{\circ} \mathrm{C} ; \mathrm{HR}=60 \% ; \mathrm{v}=1,5 \mathrm{~m} / \mathrm{s}$

\section{Conclusão}

A análise do desempenho dos controladores mostrou bons resultados. $\mathrm{O}$ controle das três componentes dentro da instalação de secagem temperatura, umidade relativa e velocidade - foi satisfatório, apresentando valores que atendem às necessidades experimentais para a determinação da cinética de secagem do bacalhau, produto para o qual a instalação foi projetada.

Destaca-se que a metodologia utilizada pode ser empregada para o controle de parâmetros de qualquer tipo de processo, não sendo restrita à secagem de produtos alimentares.

\section{Agradecimentos}

Os autores agradecem à Fundação para a Ciência e a Tecnologia - FCT, pelo apoio na condução deste trabalho.

\section{Referências Bibliográficas}

Azevedo, F.; Brasil, L. e Oliveira, R. (2000). Redes Neurais com aplicações em Controle e em sistemas Especialistas. Florianópolis: Visual Books.

Feng, G.; Lu, G.; Sun, D. and Zhou, S. (2002). A model reference adaptive control algorithm for fuzzy dynamic systems. In: 4th Congress on Intelligent Control and Automation, 3242-3246.

Gou, P.; Comaposada, J.; Serra, E.; Corominas, M.; Poch, M. and Arnau, J. (2005). Fuzzy Control System in Drying Process of Fermented Sausages. Drying Technology, 23 (9), 20552069. DOI: $10.1080 / 07373930500210523$

Murugesan, K.; Thomas, H.R. and Cleall, P.J. (2002). An investigation of the influence of twostage drying conditions on convective drying of porous materials. International Journal of Numerical Methods for Heat and Fluid Flow, 12 (1), 29-46. DOI: 10.1108/09615530210413154

Olsson, G. and Newell, B. (1999). Wastewater Treatment Systems, Modelling, Diagnosis and Control. IWA Publishing, London. 